\title{
Qualitätssicherung in der Medizin und Effektivität im Gesundheitswesen
}

\author{
Irreführende Interpretation und Hochrechnungen der Resultate von «adverse events»-Studien
}

M. Geiser

a Unter «adverse event» (AE) versteht man eine Schädigung des Patienten, verursacht durch eine Behandlung, die zur Verlängerung des Spitalaufenthaltes oder zu vorübergehender oder dauernder Belästigung oder Invalidität des Patienten zur Zeit der Spitalentlassung führt [6]. Ein AE liegt vor, wenn die Schädigung des Patienten eher durch die medizinische Behandlung als durch die Krankheit oder den Unfall entstand [7]. Ein $\mathrm{AE}$ als Folge eines Irrtums in der Planung oder Ausführung einer Behandlung wird als verhütbar beurteilt [10] im Gegensatz zu einem $\mathrm{AE}$, der nicht verhinderbar oder voraussehbar ist [8].

b Einem solchen Meldesystem kann auch das Critical Incident Reporting $[12,13]$ zugeordnet werden.

c Mit Behauptungen wie «Tausende Patienten sterben jährlich an den Folgen von Arztfehlern - oft werden die Missstände vertuscht» und Schlagzeilen wie «Russisches Roulette für Spitalpatienten» und «Götter in Weiss operieren nicht immer mit der Wahrheit» [1-3] appellierte der Bobachter an die Sensationslust des Publikums und das Sündenbockbedürfnis von Politikern.

Korrespondenz:

Prof. Dr. med. Max Geiser

Gossetstrasse 49

CH-3084 Wabern
In nordamerikanischen, australischen und englischen Spitälern wurden retrospektive Analysen [4-9] von Krankengeschichten mit Episoden von iatrogenen Schädigungen, sogenannten «adverse events» $\left(\mathrm{AEs}^{\mathrm{a}}\right)$, durchgeführt. Diese Pilotstudien in Akutspitälern zeigen weit auseinanderliegende Zahlen von AEs von 3\% in Colorado und Utah [9], 3,7\% in New York [7, 8], 10,8\% in zwei Spitälern Londons [4] und 16,6\% in Australien [5]. Diese Tatsache beweist die Schwierigkeiten bei der retrospektiven Beurteilung, ob ein AE vorlag, welche Rolle er spielte und ob ein vermeidbarer Irrtum, Nachlässigkeit oder eine unvermeidbare Komplikation vorlag. Es wurden 6 Stufen für die Rolle eines AE verwendet von «more likely» bis «virtually certain» $[4,9]$. Alle Studien fanden, dass etwa die Hälfte der AEs vermeidbar seien (Irrtum oder Nachlässigkeit bei Planung oder Ausführung der Behandlung) und dass zwei Drittel der vermeidbaren AEs keine ernsthaften Konsequenzen haben. Ausserdem zeigte sich, dass das Risiko für einen tödlichen Ausgang nach einem AE bei Patienten im höheren und hohen Alter wegen der Komplexität der Alterskrankheiten und wegen koexistierender Krankheiten doppelt so hoch ist wie bei 16- bis 44jährigen [6]. In keiner Studie wird behauptet, dass AEs die alleinige Ursache bei schweren Schäden und Tod seien. In der umfassendsten Studie (Harvard-Medical-Practice-Studie von 30121 Krankengeschichten aus dem Jahr 1984) $[7,8]$ kam es in $13,6 \%$ der $3,7 \%$ AEs zu Todesfällen «at least in part as result of $\mathrm{AE}$ ». In allen Studien wird vor einer Verallgemeinerung der Resultate der Pilotstudien aus wenigen Spitälern auf einen ganzen Staat und auf andere Länder gewarnt. Trotzdem präsentierten politisch orientierte Publikationen [10, 11] Hochrechnungen aufgrund der Pilotstudien mit entsprechenden Empfehlungen für die Qualitätssicherung im Spitalbetrieb. Das englische Gesundheitsministerium [11] empfiehlt u.a. die Schaffung eines obligatorischen und vertraulichen Meldesystems von «adverse health care events und near misses»b, die Analyse von AEs und die landes- weite Verbreitung der daraus zu ziehenden Lehren, eine Verbesserung der AE-Forschung zur Erarbeitung von zuverlässigen Daten und die Identifizierung von ernsthaften, sich wiederholenden AEs. Das englische Gesundheitsministerium ist der Ansicht [11], dass schwerwiegende AEs selten und der klinische Standard im National Health Service hoch sei. Aufgrund von Hochrechnungen kommt es in Grossbritannien jährlich zu 300000 bis 1,4 Millionen AEs, zu 2500 registrierten schwerwiegenden AEs, zu 432 schwerwiegenden AEs «involving medical devices» mit 87 Todesfällen, zu 10000 ernsthaften Arzneimittelreaktionen, zu 20000 Todesfällen innerhalb von 30 Tagen nach chirurgischen Eingriffen, zu Kosten von 1 Milliarde Pfund durch Spitalinfektionen (15\% verhütbar) und zu Kosten von 2 Milliarden Pfund für zusätzliche Spitaltage. Diese Hochrechnungen basieren z.T. auf den Ergebnissen von zwei Pilotstudien in zwei Londoner Spitälern [4] mit einem besonders risikoreichen Patientengut und ungünstigen Arbeitsverhältnissen [14]. Wenn die für Grossbritannien hochgerechneten Zahlen von AEs mit schwerwiegenden Folgen auf die 9mal kleinere Einwohnerzahl der Schweiz übertragen werden, resultiert jährlich eine Zahl von 200300 Patienten, die als Folge von medizinischen Fehlleistungen schwer geschädigt werden. Von diesen 200-300 schwerwiegenden AEs enden weniger als 100 mit dem Tod und nicht 2000-3000, wie aus dem Bundesamt für Sozialversicherung verlautete und vom Beobachter verbreitet wurde. ${ }^{c}$ Wahrscheinlich ist auch diese viel kleinere Zahl immer noch viel zu hoch und widerspiegelt die Realität nicht. Dem Bundesamt für Sozialversicherung diente offenbar die Hochrechung im Buch «To Err is Human» [10] als Grundlage, in dem für die USA 44000-98000 Todesfälle als Folge von AEs genannt werden, wobei die in den Pilotstudien dargelegte kausale Komplexität des tödlichen Ausgangs nach einem AE und die Fragwürdigkeit von Hochrechnungen nicht beachtet wurden. 


\section{Verhütungsmöglichkeiten von «adverse events»}

Niemand bezweifelt, dass ärztliche Fehlleistungen bei der Behandlung von Patienten innerhalb und ausserhalb des Spitals in allen Ländern mit mehrheitlich leichten, vorübergehenden, aber auch einzelnen schweren Schädigungen des Patienten vorkommen und beträchtliche Kosten verursachen. «Primum non nocere» ist seit den Hippokratikern eine ärztliche Pflicht im Interesse des Patienten und der Allgemeinheit (Kosten). Und es wäre verfehlt zu behaupten, dass die dank der wissenschaftlichen Fundierung der Medizin erfreulich erhöhte Patientensicherheit nicht weiter erhöht werden könne, um iatrogene Schädigungen zu vermeiden. Iatrogene Schädigungen waren in der vorwissenschaftlichen Ära der Medizin so häufig und die Effektivität der Medizin so gering, dass es für den Patienten sicherer war, die Ärzte zu meiden und der Selbstheilungskraft der menschlichen Natur zu vertrauen [15]. Wegen der zunehmenden Zahl gebrechlicher alter Patienten mit komplexen und koexistierenden Krankheiten und der Zunahme der Komplexität der möglichen reparaturmedizinischen Behandlungsmethoden und -abläufe ist die Beachtung der Pflicht der Akteure im Gesundheitswesen, nach bestem Wissen und Gewissen zu handeln und dabei die höchstmögliche Sicherheit für den Patienten zu gewährleisten, unbestritten, aber anspruchsvoller geworden.

Die Autoren der Harvard-Medical-PracticeStudie äussern die Ansicht, dass die Zahl von AEs mit Hilfe von wissenschaftlicher Forschung, Aus- und Weiterbildung und Systemanalyse vermindert werden kann. Die Systemforschung gehe über die Identifizierung schuldiger Personen hinaus. AEs entstünden durch die Interaktion von Patienten, ihrer Krankheit und einer Behandlung durch eine Gruppe von Ärzten, Pflegepersonal und weiterem Personal und der medizinischen Industrie, die Apparate und Medikamente liefert. Die in den politisch orientierten Publikationen [10, 11] erhobene These, dass «Human errors should be seen as a consequence, not a cause, of failure», beurteilt das Problem vorwiegend aus der Sicht der Systemanalyse. Ob die Übertragung der Erfahrungen mit der Qualitätssicherung in der Industrie, z. B. der Luftfahrt, auf die Medizin funktioniert, kann jedoch erst nach prospektiv durchgeführten Studien, die noch fehlen, fundiert beurteilt werden. Die Tatsache darf nicht übersehen werden, dass der wissenschaftliche Fortschritt und die Fachkompetenz, Erfahrung und Zuverlässigkeit der Ärzte und des Pflegepersonals eine ausschlaggebende Rolle für die Zunahme der Sicherheit bei der medizinischen Behandlung spielte und spielt. Die Anästhesietodesfälle beispielsweise wurden von 1:10 $000 \mathrm{im}$ Jahre 1970 auf 1:200 $000 \mathrm{im}$ Jahre 1992 reduziert [9]. Die Infektionsrate bei Operationen sank von 50\% und mehr im 19. Jahrhundert auf 1-0,1\% [16] heute. Ferner kann nicht übersehen werden, dass Qualitätssicherungsmethoden, die in einer standardisierbaren Industrie funktionieren, wegen der Individualität des Menschen und der bei weitem nicht vollkommenen Kenntnisse der Pathophysiologie des menschlichen Organismus nur auf einige, vor allem organisatorische Abläufe übertragen werden können.

Hinter der Forderung nach zentralen Meldestellen von AEs und CIs und nach einem Wechsel von der strafandrohenden Haftpflichtpraxis, die sich auf die Identifizierung schuldiger Personen konzentriert, zum «no fault compensation»System steckt möglicherweise auch die in den USA übliche enorme Höhe der Haftpflichtversicherungsprämien und Entschädigungssummen. Dieser Systemwechsel ist bei amerikanischen Anwälten umstritten [7]. Für europäische Länder mit einem niedrigeren Niveau von Haftpflichtforderungen und Versicherungsprämien ist der Ruf nach einem Systemwechsel [11, 17] nicht ohne weiteres plausibel. Denn offensichtlich bekämpft die strafandrohende Haftpflichtpraxis Inkompetenz und Nachlässigkeit der Akteure im Gesundheitswesen im Interesse des Patienten und seines Entschädigungsanspruchs und dies nicht erst seit einigen Jahren.

\section{Wie gross ist der Beitrag des «Qualitätsmanagements» zur Effektivität des Gesundheitswesens?}

Bei der Beurteilung der den Ärzten auferlegten Pflicht, den Patienten vor iatrogenen Schäden zu bewahren, stellen sich zwei Fragen. Treten heute AEs häufiger auf als in früheren Jahren? Und wie gross ist der Beitrag des «Qualitätsmanagements» des medizinischen Behandlungsablaufs zur Effektivität des Gesundheitswesens?

Für die Annahme eines gehäuften Auftretens von AEs gibt es keine beweisende Zahlen. Die Zunahme der Komplexität medizinischer Massnahmen und der Zahl gebrechlicher Patienten in hohem Alter mit komplexen und koexistierenden Krankheiten erhöhen die Möglichkeit von Komplikationen, Fehlleistungen, «adverse events» und «critical incidents» [4-9]. 
Der Beitrag der «Qualitätssicherung» des Behandlungsablaufes zur Effektivität, d.h. zur Qualität des Gesundheitswesens, wird vor allem von Laien (z. B. Politikern und Ökonomen) überschätzt, denen die Problematik von Nachweis und Kontrolle der tatsächlichen Wirksamkeit nicht vertraut ist und die irrtümlicherweise Beliebtheit und Wohlbefinden als Beweise für Wirksamkeit halten. Auf die Tatsache, dass AEs nicht notwendigerweise eine schlechte Behandlung signalisieren und dass das Fehlen von AEs nicht notwendigerweise eine gute, wirksame Behandlung bedeute, haben die Autoren der Harvard-Medical-Practice-Studie hingewiesen $[7,8]$. Selbst wenn alle vermeidbaren AEs eliminiert werden könnten, kann nicht übersehen werden, dass die grösste Beeinträchtigung der Qualität und Ökonomie eines Gesundheitswesens erfolgt, wenn effektlose diagnostische, therapeutische und präventive Massnahmen durchgeführt und an sich wirksame Massnahmen ohne oder mit falscher Indikation angewandt werden [18]. Der heutige Patient erwartet als selbstverständlich, dass medizinische Massnahmen qualitativ einwandfrei, d.h. "gegen iatrogene Schädigung gesichert», durchgeführt werden.

Der Beweis für die Wirksamkeit liegt bei vielen, aber nicht bei allen (vor allem nicht bei neuen) medizinischen Massnahmen für den vorsichtigen, kritischen medizinischen Sachverständigen auf der Hand, während die Hoffnung verängstigter Patienten und therapeutischer Übereifer zur «folie à deux» führen kann [18-22]. Diese nährt falsche Hoffnungen und bläht den Gesundheitsmarkt leerlaufend auf. Dies beeinträchtigt die Qualität und Ökonomie eines Gesundheitswesens weit mehr als die AEs, die bei erwiesen wirksamen Massnahmen aus verschiedenen vermeid- und unvermeidbaren Gründen auftreten können, aber selten auftreten. Übereifrig und leichtgläubig realisierte effektlose Massnahmen wirken ausserdem in dem Sinn iatrogen schädigend, indem sie den Patienten glauben lassen, schwerkrank und z.B. bei banalen selbstheilenden Beschwerden im Bereiche des Bewegungsapparates multidisziplinär intensiv diagnostisch und therapeutisch behandlungsbedürftig oder gar invalid zu sein. Von Medien unterstützt hat die WHO mit ihrer Gesundheitsdefinition die Gesundheitserwartungen utopisch ausgeweitet. Denn wenn der Mensch sich erst dann gesund fühlen soll, wenn er nicht nur unter keiner Krankheit («diseasefree» auf Neudeutsch) und keinen Unfallfolgen leidet, sondern das physische, psychische und soziale Wohlbefinden erreicht hat, werden die gesun- den Menschen zur Minderheit und die unheilbar Kranken, von denen viele keine unheilbare Krankheit haben [23], zur Mehrheit, die mit allen Mitteln totales Wohlbefinden fordert. Satiren über den überbordenden Gesundheits- und Wellnessmarkt drängen sich auf. Sie erscheinen spärlich [24, 25] und sind bei den Massenmedien nicht gefragt oder als allzu wahr verpönt.

\section{Zusammenfassung}

1. Die vom kausal verstehenden Denken des Naturforschers geleitete Medizin [21] liess ein Gesundheitswesen mit einer bemerkenswerten Wirksamkeit und hohen Sicherheit für den Patienten entstehen [26]. Heute stellt sich das Problem, alte und neue Torheiten und Trugschlüsse $[18,19,22]$ durch klinische Forschungsmethoden $\mathrm{zu}$ überprüfen, um eine überschiessende, unökonomische Aktivität zu vermeiden. Gesicherte Forschungsergebnisse müssen nicht nur vermehrt, sondern auch vermittelt und verbreitet und Hindernisse gegen ihre indizierte Anwendung überwunden werden [27].

2. Die bisherigen Studien über Schädigungen während der Spitalbehandlung zeigen [4-9], dass die von den Medien und vom Bundesamt für Sozialversicherung verbreiteten Zahlen über Todesfälle als Folge von Arztfehlern [1-3] masslos übertrieben sind.

3. Die Qualität eines Gesundheitswesens hängt nach wie vor vor allem von der Kompetenz, Motivation und Erfahrung der Akteure und der Respektierung gesicherter Fakten durch sie ab, um einen Schäden und Kosten provozierenden, diagnostischen, therapeutischen und präventiven Leerlauf zu vermeiden.

4. Es ist verständlich, dass ein Vertreter eines Fachbereiches, der die Sicherheit der Patienten seit Jahrzehnten vorbildlich verbessert hat [9], von den heute propagierten Qualitätssicherungsmethoden einen bürokratischen Leerlauf und eine Schädigung des Arbeitsklimas befürchtet [28]. «Qualitätsmanagement», «adverse events», «near misses» und «critical incidents» sind wie die «Evidence-based Medicine» neuzeitliche Modebegriffe [29, 30], die den Vätern und Grossvätern der heutigen Medizinergeneration unterstellen, der Wirksamkeit medizinischer Massnahmen und der Patientensicherheit als wesentliches Qualitätsmerkmal eines Behandlungsprozesses nicht die notwendige Beachtung geschenkt zu haben [12]. Sie eignen sich ausserdem auch zur Diffamierung 
der wissenschaftlich fundierten Medizin als gefährliche, nicht ganzheitliche, ergänzungsbedürftige, intolerante und nicht mehr bezahlbare «Schulmedizin» und zur Massregelung eines Berufsstandes, der die Individualität des Menschen respektiert und die vertrauensvolle Arzt-Patienten-Partnerschaft verteidigt.

5. Die Tatsache der Menschlichkeit des Irrens sollte nicht dazu verleiten, Inkompetenz, Nachlässigkeit, Unerfahrenheit und Kommunikationshindernisse resignierend als «Restrisiko» hinzunehmen und Täter vor allem als Opfer von Systemfehlern $[10,11] \mathrm{zu}$ betrachten und von der Übertragung der in der standardisierbaren Industrie entwickelten Systemanalyse auf die Medizin Wunder zu erwarten. Es darf nicht übersehen werden, dass die Fähigkeit, Irrtümer zu erkennen und daraus zu lernen, die entscheidende Voraussetzung für den Erfolg des homo sapiens bei der Erforschung der Natur darstellt [31].

\section{Literatur}

1 Haas E. Tödliche Irrtümer. Tausende Patienten sterben jährlich an den Folgen von Arztfehlern oft werden Missstände vertuscht. Beobachter Nr. 3/2001.

2 Bachmann I. Russisches Roulette für Spitalpatienten. Editorial. Beobachter Nr. 3/2001.

3 Haas E. Götter in Weiss operieren nicht immer mit der Wahrheit. Beobachter Nr. 3/2001. (diesen artikel gibt es nicht auf der website des beobachters)

4 Vincent C, Neale G, Woloshynowych M. Adverse events in British hospitals: preliminary retrospective record review. Br Med J 2001;322:517-19.

5 Wilson RM, Runciman WB, Gibberd RW, Harrison BT, Newby L, Hamilton JD. The Quality in Australian Health Care Study. Med J Aust 1995;163:458-71

6 Neale G, Woloshynowych M, Vincent C. Exploring the causes of adverse events in NHS hospital practice. J R Soc Med 2001;94:322-30.

7 Brennan TA, Leape LL, Laird NM, Hebert L, Localio AR, Lawthers AG, et al. Incidence of adverse events in hospitalized patients. Results of the Harvard Medical Practice Study I. N Engl J Med 1991;324:370-6.

8 Leape LL, Brennan TA, Laird NM, Lawthers AG, Localio AR, Barnes BA, et al. The nature of adverse events in hospitalized patients. Results of the Harvard Medical Practice Study II. N Engl J Med 1991;324:377-84.

9 Gawande AA, Thomas EJ, Zimmer MJ, Brennan TA. The incidence and nature of surgical adverse events in Colorado and Utah in 1992. Surgery 1999;126:66-75.

10 Kohn LT, et al. To Err is Human. Building a Safe Health System. Institute of Medicine. Washington DC: National Academy Press; 1999.
11 Department of Health. An organisation with a memory. Report of an expert group on learning from adverse events in the NHS. London: Department of Health; 2000.

12 Kaufmann M, Staender S, von Below G, Brunner $\mathrm{HH}$, Portenier L, Scheidegger D. Computerbasiertes anonymes Critical Incident Reporting: Ein Beitrag zur Patientensicherheit. Schweiz Ärztezeitung 2002;83:2554-8.

13 von Below G, Kuhn Hp. CIRSmedical - Update. Schweiz Ärztezeitung 2003;84:1396-8.

14 Neale C. Schriftliche Mitteilung am 3. Oktober 2001.

15 Skrabanek P. Health quackery - holding back the tide. Int J Risk Safety Med 1990;1:65-9.

16 Waugh W. John Charnley - The Man and the Hip. London: Springer; 1990.

17 Kuhn HP. Congress should pass legislation to extend protection. Schweiz Ärztezeitung 2001;82:1394-1403.

18 Cochrane AL. Effectiveness and Efficiency. Random Reflections on Health Services. Abingdon and London: The Nuffield Provincial Hospitals Trust; 1972.

19 Skrabanek P, McCormick J. Follies and Fallacies in Medicine. Glasgow: The Tarragon Press; 1989.

20 Sanitätsdirektion Baselland. Grünes Licht für Geistheilerprojekt am Bruderholzspital. Basler Zeitung vom 31.10. 2000.

21 Bleuler E. Das autistisch-undisciplinierte Denken in der Medizin und seine Ueberwindung. Berlin: Springer Verlag; 1927.

22 Geiser M. 50 Jahre Randomized Controlled Clinical Trial. Schweiz Ärztezeitung 2003;84: 598-601.

23 Klaesi J. Der unheilbar Kranke und seine Behandlung. Rektoratsrede. Bern: Paul Haupt Verlag; 1950.

24 Bösch K. Bekenntnisse meine Gesundheit betreffend. Schweiz Ärztezeitung 1999;80:2150-1.

25 Bösch K. Empowerment und 1200 für alle. Schweiz Ärztezeitung 2001;82:2154-5.

26 Thomas L. Medical lessons from history. In: Thomas L. The Medusa and the Snail. New York: The Viking Press; 1976. p. 158-75.

27 McNeil BJ. Shattuck Lecture - Hidden Barriers to improvement in quality of care. N Engl J Med 2001;345:1612-20.

28 Derron M. Kann das Qualitätsmanagement das Jahr 2004 erleben? Schweiz Ärztezeitung 2003;84:1702-3

29 Charlton BC, Miles A. The rise and fall of EBM. QJM 1998;91:371-4.

30 Geiser M. Verfehlt die «Evidence-based Medicine» das Effektivitätsziel der seriösen Medizin? Schweiz Ärztezeitung 1999;80:2717-21.

31 Thomas L. To Err is Human. In: Thomas L. The Medusa and the Snail. New York: The Viking Press; 1979. p. 36-44. 